\title{
Hydrocortisone/Pramoxine-containing Preparation
}

National Cancer Institute

\section{Source}

National Cancer Institute. Hydrocortisone/Pramoxine-containing Preparation. NCI

Thesaurus. Code C29380.

A combination preparation containing the glucocorticoid hydrocortisone acetate and the local anesthetic pramoxine hydrochloride, in aerosol foam form. Hydrocortisone has topical anti-inflammatory, vasoconstrictive and anti-pruritic activities and is used to relieve anorectal inflammation, pain, swelling, and pruritus caused by hemorrhoids. 